\title{
UPPER AND LOWER BOUNDS OF THE RADII OF GYRATION OF CONVEX BODIES $\left({ }^{1}\right)$
}

\author{
BY \\ TSUAN WU TING
}

1. Introduction. Consider a bounded convex body $K$ of dimension $n>1$. With reference to a Cartesian coordinate system, $X \equiv\left(x_{1}, x_{2}, \ldots, x_{n}\right)$, we shall denote by $V(K)$ the volume of $K, m\left(K, x_{i}\right)$ the moment of $K$ about the hyperplane $x_{i}=0$ for $i=1,2, \ldots, n, C_{K}$ the centroid of $K$, and $\bar{x}_{i}(K)$ the $i$ th coordinate of $C_{K}$. Let $\mathbf{u}$ be a unit vector emanating from $C_{K}$ such that for some positive number $\varepsilon$ the other end point of $\varepsilon \mathbf{u}$ is on the surface of a solid ball contained in $K$. We shall denote by $I\left(K, C_{K}, \mathbf{u}\right)$ or $I(K, l)$ the moment of inertia of $K$ about the line, $l\left(C_{K}, \mathbf{u}\right)$, which passes through $C_{K}$ and has the direction $\mathbf{u}$, and $g\left(K, C_{K}, \mathbf{u}\right)$ or $g(K, l)$ the radius of gyration of $K$ about the line $l\left(C_{K}, \mathbf{u}\right)$. Also, we shall use $\rho$ to denote the usual distance between two points or two sets. In particular, $\rho(X, l)$ stands for the distance from the point $X$ to the line $l$. As usual, the above kinematic quantities are defined by the following formulas:

$$
\begin{gathered}
V(K) \equiv \int_{K} d X, \quad d X=d x_{1} d x_{2} \ldots d x_{n} \\
m\left(K, x_{i}\right)=\int_{K} x_{i} d X, \quad \bar{x}_{i}(K)=m\left(K, x_{i}\right) / V(K), \quad i=1,2, \ldots, n, \\
I\left(K, C_{K}, \mathbf{u}\right)=\int_{K}\left[\rho\left(X, l\left(C_{K}, \mathbf{u}\right)\right)\right]^{2} d X \\
g\left(K, C_{K}, \mathbf{u}\right)=\left[I\left(K, C_{K}, \mathbf{u}\right) / V(K)\right]^{1 / 2}
\end{gathered}
$$

Further, for a fixed line $l\left(C_{K}, \mathbf{u}\right)$ we define

$$
\delta\left(K, C_{K}, \mathbf{u}\right)=\sup \left\{\rho\left(X, l\left(C_{K}, \mathbf{u}\right)\right): X \in K\right\} .
$$

Consider the relative radius of gyration of the convex body $K$ about the line $l\left(C_{K}, \mathbf{u}\right)$,

$$
\gamma\left(K, C_{K}, \mathbf{u}\right) \equiv g\left(K, C_{K}, \mathbf{u}\right) / \delta\left(K, C_{K}, \mathbf{u}\right) .
$$

From (1.1)-(1.3) it is clear that for a given $K, \gamma\left(K, C_{K}, \mathbf{u}\right)$ is a continuous function defined on a unit sphere of dimension $n-1$. Hence there are two directions, $\mathbf{u}_{M}(K)$ and $\mathbf{u}_{m}(K)$, such that

$$
\begin{aligned}
& \gamma\left(K, C_{K}, \mathbf{u}_{M}(K)\right)=\text { maximum of } \gamma\left(K, C_{K}, \mathbf{u}\right) \text { for all } \mathbf{u}, \\
& \gamma\left(K, C_{K}, \mathbf{u}_{m}(K)\right)=\text { minimum of } \gamma\left(K, C_{K}, \mathbf{u}\right) \text { for all } \mathbf{u} .
\end{aligned}
$$

Presented to the Society, January 27, 1967; received by the editors July 28, 1966.

(1) This work was partially supported by AFOSR, ARO, and ONR through the Joint Services Advisory Group with North Carolina State University at Raleigh; AFOSR-444-66. 
It is easy to see that the values in (1.4) and (1.5) are both independent of the volume $V(K)$. Consequently, they are domain functionals defined on all convex bodies with a given volume.

Several years ago, McShane proposed the following problem [1]: find the least upper bound and the greatest lower bound for the functionals defined in (1.4) and (1.5) respectively. For $n=3$, he showed in an elegant way that the greatest lower bound is $1 /(15)^{1 / 2}$ and conjectured that for $n=3$ the least upper bound is $1 /(2)^{1 / 2}$. The object of this work is to solve McShane's problem. In particular, his conjecture will be proved.

We shall say that a convex body $K$ is a right circular cylinder of dimension $n>1$, if it is the Cartesian product of an interval with a solid ball of dimension $n-1$. Thus, in space $E_{3}$ it is an ordinary right circular cylinder and in space $E_{2}$ it is a rectangle. After introducing the above definitions, we now state the results as a

THEOREM. If $K$ is a bounded convex body of dimension $n \geqq 2$, then

$$
\begin{aligned}
& {\left[\gamma\left(K, C_{K}, \mathbf{u}_{M}(K)\right)\right]^{2} \leqq(n-1) /(n+1), } \\
& {\left[\gamma\left(K, C_{K}, \mathbf{u}_{m}(K)\right)\right]^{2} } \geqq 1 / 8 \text { for } n=2, \\
&>1 / n(n+2) \text { for } n>2 .
\end{aligned}
$$

The equality sign in (1.6) holds only when $K$ is a right circular cylinder; while for all integers $n>2$ the numbers in (1.7) are the greatest lower bounds and for $n=2$ the equality sign in (1.7) holds only when $K$ is a right triangle.

In what follows, we shall present a proof for the inequality (1.6), the first inequality in (1.7) and a slight modification of McShane's technique for proving the second inequality in (1.7). Analogous to the technique adopted in [2], the proof is to compare a given convex body with the solution figure which was conjectured in advance. However, it is difficult to make such a comparison directly. Accordingly, the proof is to develop a comparison scheme by constructing intermediate bodies.

2. Lemmas. In this section we shall establish a few lemmas which will play very important roles in the proof of the theorem. The first lemma is an estimate of the higher-order radius of gyration of a two-dimensional "cone-like" convex body.

LEMMA 1. Let $f(x)$ be a bounded nonnegative concave function defined for $0 \leqq x \leqq x_{1}$. Let $K$ be the plane convex set which is bounded from the left, from the right and from below by the lines $x=0, x=x_{1}, y=0$ respectively and bounded from above by the curve,

$$
y=f(x), \quad 0 \leqq x \leqq x_{1} .
$$

For every integer $n \geqq 2$, we define

$$
m_{n-1}(K) \equiv \int_{0}^{x_{1}} x^{n-1} f(x) d x, \quad x^{*}(K, n-1) \equiv m_{n-1}(K) / m_{n-2}(K) .
$$


Then

$$
x_{1}(n-1) /(n+1) \leqq x^{*}(K, n-1) \leqq n x_{1} /(n+1) .
$$

Proof. First, we consider the simple case,

$$
f(x)=\alpha x, \quad \alpha \text { is a positive number. }
$$

We readily compute that in this case $x^{*}(K, n-1)$ is equal to $n x_{1} /(n+1)$. Secondly, consider the case

$$
f(x)=y_{0}\left(1-x / x_{1}\right), \quad y_{0} \text { is a positive constant. }
$$

Again, direct computation gives that $x^{*}(K, n-1)$ is equal to $x_{1}(n-1) /(n+1)$. Thus, the lemma is true for these two right triangles.

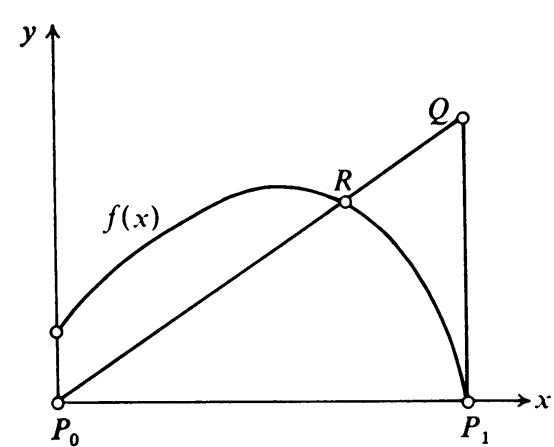

FIGURE 1a

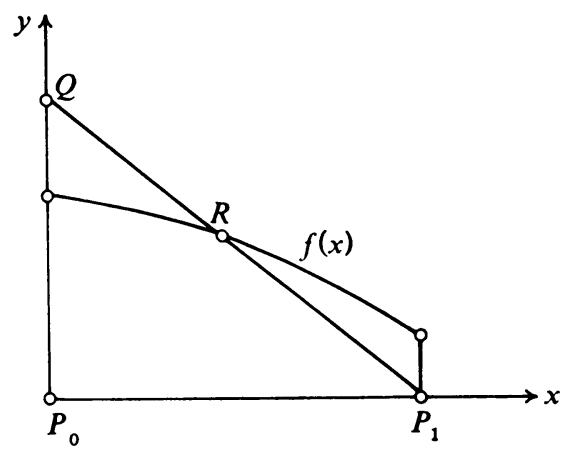

FIGURE $1 \mathrm{~b}$

Suppose that $K$ is not a right triangle with its base on the $x$-axis. Denote by $P_{0}, P_{1}$ the points with coordinates $(0,0)$ and $\left(x_{1}, 0\right)$ respectively. Consider a triangle $\Delta$ with its vertices at $P_{0}, P_{1}$ and $Q$ such that the segment $P_{1} Q$ is perpendicular to the $x$-axis and the point $Q$ lies in the upper-half plane (see Figure 1a). Since the segment $P_{0} Q$ is defined by $y=\alpha x$, we may choose the positive constant $\alpha$ to be such that

$$
m_{n-2}(\Delta) \equiv \int_{0}^{x_{1}} \alpha x^{n-1} d x=\int_{0}^{x_{1}} x^{n-2} f(x) d x \equiv m_{n-2}(K) .
$$

With such a choice of $\alpha$, the point $Q$ must stay in the complement of $\bar{K}$, the closure of $K$. Otherwise, we will have a contradiction to (2.4), because $K$ is, by hypothesis, not a right triangle. Since the point $Q$ is in the complement of $\bar{K}$, there is a disk $D$ containing $Q$ and contained in the complement of $\bar{K}$. The area of the intersection of the disk $D$ with the triangle $\Delta$ is obviously positive. Thus, if the segment $P_{0} Q$ does not intersect the interior of $K$, then we have a contradiction to (2.4) again, because the segment $P_{1} R$ cannot meet the interior of $K$. Hence the segment $P_{0} Q$ must be divided by $\partial K$, boundary of $K$, into two segments $P_{0} R$ and $R Q$ such that the open segment $P_{0} Q$ is contained in $K-\partial K$ and the open segment $R Q$ is contained in the complement of $\bar{K}$ (see Figure 1a). 
Denote by $(\hat{x}, \hat{y})$ the coordinates of the point $R$. Then $0<\hat{x}<x_{1}$ and we have

$$
m_{n-1}(\Delta)-m_{n-1}(K)=\int_{0}^{\hat{x}} x^{n-1}[\alpha x-f(x)] d x+\int_{\hat{x}}^{x_{1}} x^{n-1}[\alpha x-f(x)] d x .
$$

Since the first integrand in (2.5) must be nonpositive while the second in (2.5) must be nonnegative, we can apply the mean-value theorem for integrals to write

$$
m_{n-1}(\Delta)-m_{n-1}(K)=c_{1} \int_{0}^{\hat{x}} x^{n-2}[\alpha x-f(x)] d x+c_{2} \int_{\hat{x}}^{x_{1}} x^{n-2}[\alpha x-f(x)] d x,
$$

where the constants $c_{1}$ and $c_{2}$ satisfy the following strict inequalities,

$$
0<c_{1}<\hat{x}<c_{2}<x_{1} \text {. }
$$

But $\alpha$ was determined by the equation (2.4), so we immediately conclude from (2.6) and (2.7) that $m_{n-1}(\Delta)>m_{n-1}(K)$. This fact together with the equation (2.4) and the definition for $x^{*}$ in (2.2) shows that

$$
x^{*}(K, n-1)<x^{*}(\Delta, n-1)=n x_{1} /(n+1),
$$

and the second inequality in (2.3) is proved. The proof also shows that the second equality sign in (2.3) holds when, and only when, $K$ is a right triangle.

The first inequality in (2.3) can be proved by considering a triangle $P_{0} P_{1} Q$ with the point $Q$ on the positive $y$-axis, (see Figure $1 \mathrm{~b}$ ). Since the analysis is entirely similar to what has been shown, the details can be omitted. The proof is now complete.

LEMMA 2. For every plane convex set $K$ as specified in Lemma 1 , there is a unique trapezoid $T$ which is bounded from the left, from the right and from below by the lines $x=0, x=x_{1}$ and $y=0$ respectively and which is bounded from above by the segment

$$
y=y_{0}+\alpha x, \quad 0 \leqq x \leqq x_{1},
$$

such that

$$
\begin{gathered}
y_{0} \geqq f(0), \quad y_{0}+\alpha x_{1} \geqq f\left(x_{1}\right), \\
m_{n-2}(T)=m_{n-2}(K), \quad m_{n-1}(T)=m_{n-1}(K),
\end{gathered}
$$

where $n$ is an integer greater than or equal to two.

Proof. We show that the constants $y_{0}$ and $\alpha$ in (2.8) can be so chosen that all the requirements in (2.9) and (2.10) will be satisfied. Indeed,

$$
\begin{aligned}
& m_{n-2}(T) \equiv \int_{0}^{x_{1}} x^{n-2}\left(y_{0}+\alpha x\right) d x=m_{n-2}(K), \\
& m_{n-1}(T) \equiv \int_{0}^{x_{1}} x^{n-1}\left(y_{0}+\alpha x\right) d x=m_{n-2}(K) .
\end{aligned}
$$


Upon solving these two equations for $y_{0}$ and $\alpha$, we have

$$
\begin{aligned}
y_{0} & =n(n-1)\left[n x_{1}-(n+1) x^{*}(K, n-1)\right] m_{n-2}(K) / x_{1}^{n}, \\
\alpha & =n(n+1)\left[n x^{*}(K, n-1)-(n-1) x_{1}\right] m_{n-2}(K) / x_{1}^{n+1},
\end{aligned}
$$

where $x^{*}(K, n-1)$ is defined by the second equation in (2.2). This shows that the trapezoid $T$ is unique if it exists.

To complete the proof we verify that if the values of $y_{0}$ and $\alpha$ are given by the expressions in (2.11) then the inequalities in (2.9) are satisfied. To this end, we note that

$$
y_{0} \geqq 0 .
$$

Indeed, this follows directly from the second inequality in (2.3) and the positivity of $m_{n-2}(K)$. Furthermore, simple computation gives that

$$
y_{0}+\alpha x_{1}=n\left[(n+1) x^{*}(K, n-1)-(n-1) x_{1}\right] m_{n-2}(K) / x_{1}^{n} \geqq 0,
$$

in view of the first inequality in (2.3) and the positivity of $m_{n-2}(K)$.

To establish the inequality in (2.9) we observe that if $K$ is really a trapezoid $T$ with all the properties stated in Lemma 2 then there is nothing to prove. Accordingly, we assume that this is not the case and proceed to show that the strict inequalities in (2.9) hold.

First, suppose that

$$
y_{0} \leqq f(0) \text { and } y_{0}+\alpha x_{1} \leqq f\left(x_{1}\right) .
$$

Then the inequalities in (2.12) and (2.13) together with the convexity of $K$ imply that $T \subset K$. Since $K$ is not a trapezoid, we must have the strict inequality,

$$
m_{n-2}(T)<m_{n-2}(K),
$$

which contradicts the first equation in (2.10). Secondly, suppose that

$$
y_{0} \leqq f(0) \text { and } y_{0}+\alpha x_{1}>f\left(x_{1}\right) .
$$

Denote by $P_{0}, P_{1}, Q, R$ the points with coordinates $(0,0),\left(x_{1}, 0\right),\left(x_{1}, y_{0}+x_{1}\right)$ and $\left(0, y_{0}\right)$ respectively, (see Figure 2a). For reasons similar to the ones stated before,

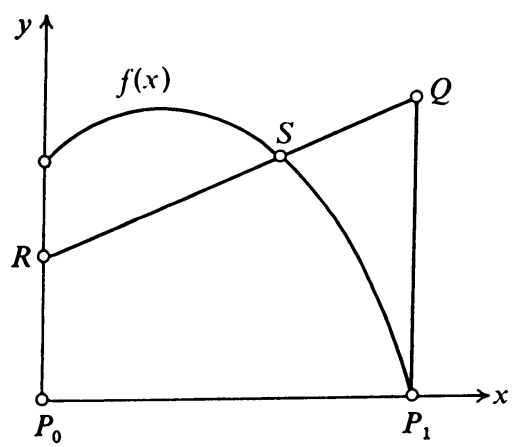

Figure 2a

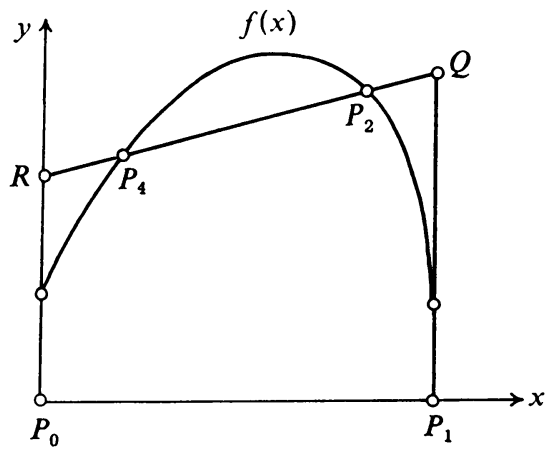

FIGURE 2b 
the segment $R Q$ must be divided by the curve $y=f(x)$ into segments $R S$ and $S Q$ such that the open segment $R S$ is contained in $K-\partial K$ and the open segment $S Q$ is contained in the complement of $\bar{K}$. Otherwise, we will immediately have a contradiction with the equations in (2.10). Denote by $(\hat{x}, \hat{y})$ the coordinates of the point $S$. Then, what has been said means that

$$
y_{0}+\alpha x<f(x), \quad 0<x<\hat{x} ; \quad y_{0}+\alpha x>f(x), \quad \hat{x}<x \leqq x_{1} .
$$

Thus we may apply the mean-value theorem to the integrals,

$$
m_{n-1}(T)-m_{n-1}(K)=\left\{\int_{0}^{\hat{x}}+\int_{\hat{x}}^{x_{1}}\right\}\left[x^{n-1}\left(y_{0}+\alpha x-f(x)\right)\right] d x,
$$

so as to obtain

$$
\begin{aligned}
m_{n-1}(T)-m_{n-1}(K) & =\left\{c_{1} \int_{0}^{\hat{x}}+c_{2} \int_{\hat{x}}^{x_{1}}\right\}\left[x^{n-2}\left(y_{0}+\alpha x-f(x)\right)\right] d x, \\
0 & <c_{1}<\hat{x}<c_{2}<x_{1} .
\end{aligned}
$$

But the trapezoid $T$ was so constructed that

$$
\left\{\int_{0}^{\hat{x}}+\int_{\hat{x}}^{x_{1}}\right\}\left[x^{n-2}\left(y_{0}+\alpha x-f(x)\right)\right] d x=0 .
$$

We conclude from (2.16)-(2.18) that $m_{n-1}(T)>m_{n-1}(K)$, which contradicts the second equation in (2.10). Completely similar reasoning shows that the case

$$
y_{0}>f(0) \text { and } y_{0}+x_{1} \leqq f\left(x_{1}\right)
$$

also cannot happen.

Since the cases (2.14), (2.15) and (2.19) all cannot happen, the two strict inequalities in (2.9) must hold simultaneously. This proof also shows that if one of the equality signs in (2.9) holds, then $K$ must be the trapezoid $T$ and hence the other equality sign in (2.9) must also hold.

The purpose to construct the trapezoid $T$ in Lemma 2 is that it has the same base as that of the "cone-like" convex set $K$ and that it has the following special properties which just suit our need.

Lemma 3. Let $K$ be the plane convex set specified in Lemma 1 and let $T$ be the trapezoid constructed in Lemma 2. Then

$$
m_{n-1+j}(T) \geqq m_{n-1+j}(K) \text { for } j=1,2,
$$

where the equality sign holds only when $K$ and $T$ are identical.

Proof. To prove the inequalities in (2.20) we again assume that $K$ is not a trapezoid $T$ so that the strict inequalities in (2.9) hold. Using the same notations as adopted in Lemma 2, we denote by $P_{0}, P_{1}, Q$ and $R$ the vertices of $T$ (see Figure 2b). If the segment $Q R$ lies in the complement of $\bar{K}$ or if it just touches $\partial K$, then we will have a contradiction to the two equations in (2.10), because the strict inequalities in (2.9) hold. Thus the segment $Q R$ has a nonempty intersection with 
$K-\partial K$. Hence the convexity of $K$ implies that $\partial K$ divides the segment $Q R$ into three segments $Q P_{2}, P_{2} P_{4}$ and $P_{4} R$ such that the open segments $Q P_{2}, P_{4} R$ are both contained in the complement of $\bar{K}$ and that the open segment $P_{2} P_{4}$ is contained in $K-\partial K$.

Denote by $x_{2}$ and $x_{4}$ respectively the abscissas of the points $P_{2}$ and $P_{4}$. Then we have

$$
\begin{gathered}
y_{0}+\alpha x>f(x) \text { for } 0 \leqq x<x_{4}, x_{2}<x \leqq x_{1}, \\
y_{0}+\alpha x<f(x) \text { for } x_{4}<x<x_{2} .
\end{gathered}
$$

Hence, for sufficiently small positive $\varepsilon$,

$$
\begin{aligned}
& \int_{0}^{x_{4}+\varepsilon} x^{n-2}\left[y_{0}+\alpha x-f(x)\right] d x>0, \\
& \int_{0}^{x_{2}-\varepsilon} x^{n-2}\left[y_{0}+\alpha x-f(x)\right] d x<0,
\end{aligned}
$$

in view of the first equation in (2.10). Consequently, from the continuity of an indefinite integral as a function of its upper limit it follows that there is a number $x_{3}, \quad x_{4}<x_{3}<x_{2}$, such that

$$
\begin{aligned}
& \int_{0}^{x_{3}} x^{n-2}\left[y_{0}+\alpha x-f(x)\right] d x=0 \\
& \int_{x_{3}}^{x_{1}} x^{n-2}\left[y_{0}+\alpha x-f(x)\right] d x=0 .
\end{aligned}
$$

Consider the value of the integral

$$
\begin{aligned}
J_{1} & \equiv \int_{0}^{x_{1}} x^{n-2}\left(x-x_{3}\right)^{2}\left[y_{0}+\alpha x-f(x)\right] d x \\
& =\left\{\int_{0}^{x_{4}}+\int_{x_{4}}^{x_{3}}+\int_{x_{3}}^{x_{2}}+\int_{x_{2}}^{x_{1}}\right\}\left[x^{n-2}\left(x-x_{3}\right)^{2}\left(y_{0}+\alpha x-f(x)\right)\right] d x .
\end{aligned}
$$

Since the inequalities in (2.21) assure that the integrand

$$
x^{n-2}\left[y_{0}+\alpha x-f(x)\right]
$$

of each of the above integrals remains the same sign throughout the respective intervals of integration, we may apply the mean-value theorem to write

$$
\begin{aligned}
J_{1} \equiv\left\{\left(c_{4}-x_{3}\right)^{2} \int_{0}^{x_{4}}+\right. & \left(c_{3}-x_{3}\right)^{2} \int_{x_{4}}^{x_{3}}+\left(c_{2}-x_{3}\right)^{2} \int_{x_{3}}^{x_{2}} \\
& \left.+\left(c_{1}-x_{3}\right)^{2} \int_{x_{2}}^{x_{1}}\right\}\left[x^{n-2}\left(y_{0}+\alpha x-f(x)\right)\right] d x,
\end{aligned}
$$

with

$$
0<c_{4}<x_{4}<c_{3}<x_{3}<c_{2}<x_{2}<c_{1}<x_{1} .
$$


The above inequalities imply that

$$
\left(c_{4}-x_{3}\right)^{2}>\left(c_{3}-x_{3}\right)^{2}, \quad\left(c_{1}-x_{3}\right)^{2}>\left(c_{2}-x_{3}\right)^{2} .
$$

Thus we conclude from (2.21)-(2.24) that the sum of the first two integrals and the sum of the last two integrals in (2.23) are both positive. Hence

$$
J_{1} \geqq 0 \text { and }=0 \text { only when } K=T \text {. }
$$

On the other hand, we have from the two equations in (2.10) that

$$
\begin{aligned}
J_{1} & =\int_{0}^{x_{1}} x^{n-2}\left(x^{2}-2 x_{3} x+x_{3}^{2}\right)\left[y_{0}+\alpha x-f(x)\right] d x \\
& =m_{n}(T)-m_{n}(K) .
\end{aligned}
$$

Now the inequality in (2.20) for $j=1$ follows directly from (2.25) and (2.26).

To establish (2.20) for $j=2$, we note from the second equation in (2.10) that there is a number $\bar{x}_{3}, \quad x_{4}<\bar{x}_{3}<x_{2}$, such that

$$
\begin{aligned}
& \int_{0}^{x_{3}} x^{n-1}\left[y_{0}+\alpha x-f(x)\right] d x=0, \\
& \int_{x_{3}}^{x_{1}} x^{n-1}\left[y_{0}+\alpha x-f(x)\right] d x=0 .
\end{aligned}
$$

For reasons exactly like that for deriving (2.25), we have

$$
J_{2} \equiv \int_{0}^{x_{1}} x^{n-1}\left(x-\bar{x}_{3}\right)^{2}\left[y_{0}+\alpha x-f(x)\right] d x \geqq 0,
$$

where the equality sign holds when, and only when, $K=T$. On the other hand, the second equation in (2.10) implies that

$$
J_{2}=m_{n+1}(T)-m_{n+1}(K)-2 \bar{x}_{3}\left[m_{n}(T)-m_{n}(K)\right] .
$$

Combining (2.27) and (2.28), we have, if $K \neq T$,

$$
m_{n+1}(T)-m_{n+1}(K)>2 \bar{x}_{3}\left[m_{n}(T)-m_{n}(K)\right]>0 .
$$

This also proves that the equality sign in (2.20) holds for $j=2$ only when $K=T$. The proof of Lemma 3 is complete.

It is easy to show that both the convexity of $K$ and the fact that the line $l\left(C_{K}, \mathbf{u}\right)$ passes through the centroid of $K$ are necessary for the theorem to be true. Accordingly, a proof of the theorem must use both of these two conditions. The convexity of $K$ will be used in the present proof through Lemmas 1, 2 and 3; while a global property of the centroid of a "cone-like" convex body will be used through the following lemma, which is a proper specialization and extension of Jensen's inequality [6], [8]. To simplify the statement of the next lemma we introduce the 
Definition. A bounded $n$-dimensional convex body $K$ is said to be cone-like if, relative to a Cartesian coordinate system $\left(x_{1}, x_{2}, \ldots, x_{n}\right)$, it is bounded from below by the hyperplane $x_{n}=0$ and bounded from above by the concave hypersurface.

$$
x_{n}=f\left(x_{1}, x_{2}, \ldots, x_{n-1}\right), \quad\left(x_{1}, x_{2}, \ldots, x_{n-1}\right) \in D,
$$

where $D$ is a convex body of dimension $n-1$ and $f$ is a (continuous) nonnegative, concave function of the $n-1$ independent variables, $x_{1}, x_{2}, \ldots, x_{n-1}$.

If we write

$$
\begin{gathered}
\hat{X}=\left(x_{1}, x_{2}, \ldots, x_{n-1}\right), \quad d \hat{X}=d x_{1} d x_{2} \ldots d x_{n-1}, \\
V(D)=\int_{D} d \hat{X}, \quad \bar{f}=V(K) / V(D),
\end{gathered}
$$

then it follows from the expression (2.29) and the definition in (1.1) that for $i=1,2, \ldots, n-1$

$$
m\left(K, x_{i}\right)=\int_{D} x_{i} f(\hat{X}) d \hat{X}, \quad \bar{x}_{i}=m\left(K, x_{i}\right) / V(K)
$$

LEMMA 4. If $K$ is a bounded n-dimensional cone-like convex body, then the height of $K$ at its centroid is greater than or equal to its mean height, i.e.,

$$
f\left(\bar{x}_{1}, \bar{x}_{2}, \ldots, \bar{x}_{n-1}\right) \geqq \bar{f} .
$$

Furthermore, these two heights of $K$ are equal, if and only if $K$ is a right cylinder.

3. Proof of the theorem for $n=2$. Let $H$ be a given bounded convex set in the plane. We shall first establish the inequality (1.6) for $H$. To this end, we choose $C_{H}$ as the origin of a Cartesian coordinate system such that the $y$-axis is the line $l\left(C_{H}, \mathbf{u}_{M}(H)\right)$. Now we apply the Steiner symmetrization process [3], [4] to the convex set $H$ with respect to the $x$-axis. This transforms $H$ into a set $H^{*}$. The well-known properties of Steiner symmetrization ensure that $H^{*}$ is convex, $V\left(H^{*}\right)=V(H)$ and $C_{H^{*}}$ and $C_{H}$ coincide. For simplicity, we shall write $\pi / 2$ instead of $\mathbf{u}_{M}(H)$. Then it is easy to see that

$$
I\left(H^{*}, C_{H^{*}}, \pi / 2\right)=I\left(H, C_{H}, \pi / 2\right), \quad \delta\left(H^{*}, C_{H^{*}}, \pi / 2\right)=\delta\left(H, C_{H}, \pi / 2\right) .
$$

Thus, to establish the inequality (1.6) for $H$, it suffices to show that

$$
\left[\gamma\left(H^{*}, C_{H^{*}}, \pi / 2\right)\right]^{2} \leqq 1 / 3
$$

Since the value of $\gamma\left(H^{*}, C_{H^{*}}, \pi / 2\right)$ remains unchanged under translations of the $y$-axis to the right, we may assume that the $y$-axis is a line of support of $H^{*}$. Denote by $K$ the upper half of $H^{*}$. Then we need only to show that

$$
\left[\gamma\left(K, C_{K}, \pi / 2\right)\right]^{2} \leqq 1 / 3
$$


Now, the convex set $K$ in (3.2) has all the properties as stated in Lemma 1. By Lemma 2, there is a trapezoid $T$ such that $C_{T}$ and $C_{K}$ are on the same vertical line and such that

$$
V(T)=V(K), \quad \delta\left(T, C_{T}, \pi / 2\right)=\delta\left(K, C_{K}, \pi / 2\right) .
$$

Furthermore, by Lemma 3 and by the parallel-axis theorem for moment of inertia, we have

$$
I\left(T, C_{T}, \pi / 2\right) \geqq I\left(K, C_{K}, \pi / 2\right) .
$$

Thus, to establish (3.2) it suffices to show that

$$
\left[\gamma\left(T, C_{T}, \pi / 2\right)\right]^{2} \leqq 1 / 3 .
$$

The trapezoid $T$ is uniquely determined by the equation in (2.8). For convenience, we may write it in the form:

$$
y=y_{0}+\left(y_{1}-y_{0}\right) x / x_{1}, \quad 0 \leqq x \leqq x_{1} .
$$

Moreover, from (2.3) and (2.11)-(2.i3) we see that the values of $y_{0}$ and $y_{1}$ in (3.4) must be such that either

$$
y_{0} \geqq 0, \quad y_{1}>0 \text { or } y_{0}>0, \quad y_{1} \geqq 0 .
$$

Thus, we can appeal to computation to obtain:

$$
\begin{gathered}
V(T)=\frac{1}{2}\left(y_{0}+y_{1}\right) x_{1}, \quad \bar{x}(T)=x_{1}\left(y_{0}+2 y_{1}\right) / 3\left(y_{0}+y_{1}\right), \\
I\left(T, C_{T}, \pi / 2\right)=x_{1}^{3}\left(y_{0}^{2}+4 y_{0} y_{1}+y_{1}^{2}\right) / 36\left(y_{0}+y_{1}\right) .
\end{gathered}
$$

If $y_{0} \geqq y_{1}$, then $\bar{x}(T) \geqq\left(x_{1}-\bar{x}(T)\right)$. Hence, in this case,

$$
\left[\gamma\left(T, C_{T}, \pi / 2\right)\right]^{2}=\frac{1}{2}\left\{1-3\left[y_{1} /\left(y_{0}+2 y_{1}\right)\right]^{2}\right\} \leqq 1 / 3 .
$$

On the other hand, if $y_{0} \leqq y_{1}$, then $\left(x_{1}-\bar{x}(T)\right) \geqq \bar{x}(T)$ and, in this case

$$
\left[\gamma\left(T, C_{T}, \pi / 2\right)\right]^{2}=\frac{1}{2}\left\{1-3\left[y_{0} /\left(2 y_{0}+y_{1}\right)\right]^{2}\right\} \leqq 1 / 3 .
$$

Clearly, the equality sign in (3.5) or in (3.6) holds if and only if $T$ is a rectangle. Consequently, the equality sign in (1.6) holds if and only if $K$ is a rectangle. The proof of (1.6) for $n=2$ is now complete.

We proceed to establish the inequality (1.7) for $n=2$. Let $H$ be a given bounded plane convex set. We choose $C_{H}$ as the origin of a Cartesian coordinate system with the $y$-axis being the line $l\left(C_{H}, \mathbf{u}_{m}(H)\right)$. Again, we transform $H$ into a convex set $H^{*}$ by the Steiner symmetrization process with respect to the $x$-axis. Let $K$ be the upper half of $H^{*}$. Then by the same reasoning as shown above, it suffices to show that

$$
\left[\gamma\left(K, C_{K}, \pi / 2\right)\right]^{2} \geqq 1 / 8,
$$

where we have written $\pi / 2$ to stand for the direction $\mathbf{u}_{m}(H)$. Also, without changing the value of the expression in (3.7), we may translate the $y$-axis to the right so that it becomes a line of support of $K$. 
Denote by $P_{0}, P_{1}$ the end points of the base of $K$. Their coordinates are given by $(0,0)$ and $\left(x_{1}, 0\right)$ respectively. Consider a triangle $\Delta$ with the segment $P_{0} P_{1}$ as its base and with its third vertex $Q$ in the upper-half plane (see Figure 3). Clearly, the point $Q$ can be so chosen that $V(\Delta)=V(K)$. Furthermore, it follows from Lemma 1 that if $Q$ on the line $x=x_{1}$ then $\bar{x}(\Delta) \geqq \bar{x}(K)$ and if $Q$ on the $y$-axis then $\bar{x}(\Delta) \leqq \bar{x}(K)$. Thus, from the continuity of $\bar{x}(\Delta)$ as a function of the position of $Q$, we conclude that the point $Q$ can be so chosen that $\bar{x}(\Delta)=\bar{x}(K)$. That is, $C_{\Delta}$ and $C_{K}$ are on the same vertical line.

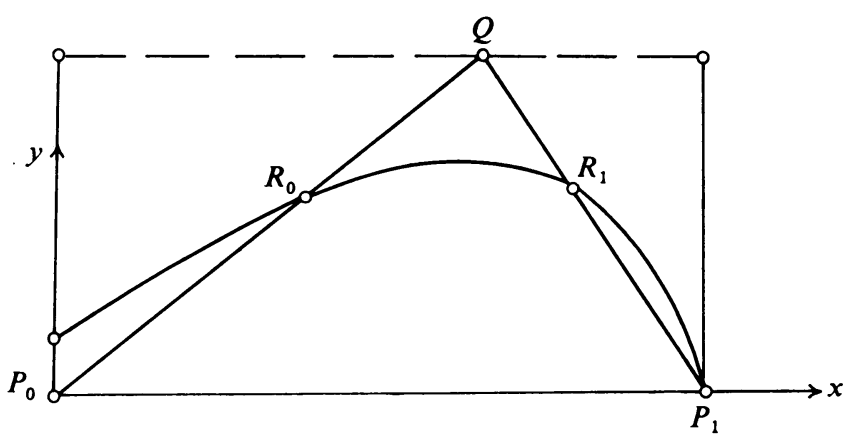

FIGURE 3

Suppose that $K$ is not a triangle. Then, the point $Q$ must stay in the complement of $\bar{K}$. If both the segments $P_{0} Q$ and $P_{1} Q$ do not meet $K-\partial K$, then $V(\Delta)>V(K)$; if one of these two segments does not meet $K-\partial K$ then either $\bar{x}(\Delta)>\bar{x}(K)$ or $\bar{x}(\Delta)<\bar{x}(K)$. Thus it follows, in view of the convexity of $K$, that the segments $P_{0} Q$ and $P_{1} Q$ meet $\partial K$ at points $R_{0}$ and $R_{1}$ respectively such that $\rho\left(P_{0}, R_{0}\right)>0$, $\rho\left(P_{1}, R_{1}\right)>0$ and such that the open segments $P_{0} R_{0}$ and $P_{1} R_{1}$ are contained in $K-\partial K$ and the open segments $Q R_{0}, Q R_{1}$ are contained in the complement of $\bar{K}$. Now, following the same reasoning as shown in the proof of Lemma 3, we conclude that $m_{2}(\Delta) \leqq m_{2}(K)$. This fact together with the parallel-axis theorem for moment of inertia implies that

$$
I\left(\Delta, C_{\Delta}, \pi / 2\right) \leqq I\left(K, C_{K}, \pi / 2\right),
$$

where the equality sign holds only when $K$ is a triangle $\Delta$. Since $\delta\left(\Delta, C_{\Delta}, \pi / 2\right)$ $=\delta\left(K, C_{K}, \pi / 2\right)$ by the method for constructing $\Delta$, to prove the inequality (3.7) it suffices to show that

$$
\left[\gamma\left(\Delta, C_{\Delta}, \pi / 2\right)\right]^{2} \geqq \frac{1}{8} .
$$

To establish (3.8), we denote by $\left(x_{2}, y_{2}\right)$ the coordinates of the point $Q$. Then segments $P_{0} Q$ and $P_{1} Q$ are respectively given by

$$
\begin{aligned}
& y=y_{2} x / x_{2}, \quad 0 \leqq x \leqq x_{2}, \\
& y=y\left[1-\left(x-x_{2}\right) /\left(x_{1}-x_{2}\right)\right], \quad x_{2} \leqq x \leqq x_{1} .
\end{aligned}
$$


Thus, by direct computation, we find

$$
\begin{gathered}
V(\Delta)=\frac{1}{2} x_{1} y_{2}, \quad \bar{x}(\Delta)=\frac{1}{3}\left(x_{1}+x_{2}\right), \\
I\left(\Delta, C_{\Delta}, \pi / 2\right)=x_{1} y_{2}\left(x_{1}^{2}-x_{1} x_{2}+x_{2}^{2}\right) .
\end{gathered}
$$

If $\bar{x}(\Delta) \geqq x_{1} / 2$, then $\delta\left(\Delta, C_{\Delta}, \pi / 2\right)=\bar{x}(\Delta)$ and, in this case,

$$
\left[\gamma\left(\Delta, C_{\Delta}, \pi / 2\right)\right]^{2}=\frac{1}{2}\left\{1-3\left(x_{2} / x_{1}\right) /\left(1+x_{2} / x_{1}\right)^{2}\right\}
$$

But $\frac{1}{2} \leqq x_{2} / x_{1} \leqq 1$ in this case and

$$
(d / d \lambda)\left[\lambda /(1+\lambda)^{2}\right]>0 \quad \text { for } \lambda<1 .
$$

Hence, in this case, (3.8) is indeed valid and the equality sign holds only when $\Delta$ is a right triangle. On the other hand, if $\bar{x}(\Delta) \leqq 1 / 2$, then $\delta\left(\Delta, C_{\Delta}, \pi / 2\right)=x_{1}-\bar{x}(\Delta)$ and, in this case,

$$
\left[\gamma\left(\Delta, C_{\Delta}, \pi / 2\right)\right]^{2}=\frac{1}{2}\left\{1-3\left(1-x_{2} / x_{1}\right) /\left(2-x_{2} / x_{1}\right)^{2}\right\} .
$$

But $0 \leqq x_{2} / x_{1} \leqq 1 / 2$ in this case and

$$
(d / d \lambda)\left[(1-\lambda) /(2-\lambda)^{2}\right]<0 \quad \text { for } 0<\lambda \leqq 1 / 2 .
$$

Hence, (3.8) is also valid in this case and the equality sign holds only when $\Delta$ is a right triangle. This proves the inequality (1.7) for $n=2$.

4. Proof of the inequality (1.6) for $n \geqq 3$. Let $Y$ be a right circular cylinder of dimension $n \geqq 3$. More specifically, relative to a Cartesian coordinate system, it is given by the formula,

$$
Y=\left\{X \mid \sum_{i=1}^{n-1}\left(x_{i}\right)^{2}=1,0 \leqq x_{n} \leqq 1\right\} .
$$

From (4.1) and (1.1) it is easy to see that $C_{Y}$ is on the $x_{n}$-axis. To compute its relative radius of gyration about the $x_{n}$-axis, we introduce a cylindrical coordinate system $\left(r, \theta_{1}, \theta_{2}, \ldots, \theta_{n-2}, x_{n}\right)$,

where

$$
\begin{aligned}
x_{n} & =x_{n}, \\
x_{1} & =r \cos \theta_{1}, \\
x_{2} & =r \sin \theta_{1} \cos \theta_{2}, \\
\cdot & \cdot \cdot \cdot \cdot \cdot \cdot \cdot \cdot \cdot \cdot \cdot \cdot \cdot \cdot \\
x_{n-2} & =r \sin \theta_{1} \sin \theta_{2} \ldots \sin \theta_{n-3} \cos \theta_{n-2} \\
x_{n-1} & =r \sin \theta_{1} \sin \theta_{2} \ldots \sin \theta_{n-3} \sin \theta_{n-2}
\end{aligned}
$$

$$
0<\theta_{n-2}<2 \pi, \quad 0<\theta_{i}<\pi, \quad i=1,2, \ldots, n-3 .
$$

For simplicity we shall write $I\left(Y, x_{n}\right), g\left(Y, x_{n}\right), \delta\left(Y, x_{n}\right)$ for the moment of inertia, the radius of gyration and the maximum radius of $Y$ with respect to the $x_{n}$-axis. 
Similar notation will also be used later on for other bodies. Then, direct computation gives

$$
\left[\gamma\left(Y, x_{n}\right)\right]^{2}=(n-1) /(n+1) .
$$

Thus the equality in (1.6) is attained by right circular cylinders with dimension greater than one.

Let $H$ be a bounded convex body with dimension $n \geqq 3$. We proceed to show that if $H$ is not a right circular cylinder, then the strict inequality in (1.6) holds for $H$. To this end, we choose a cylindrical coordinate system, (4.2), with the origin at $C_{H}$ and with the $x_{n}$-axis being the line $l\left(H, C_{H}, \mathbf{u}_{M}(H)\right)$. By applying the Steiner symmetrization process to $H$ with respect to the hyperplane $x_{n}=0$, we construct a convex body $H^{*}$ with the following properties:

(i) $C_{H^{*}}$ is at the origin, $V\left(H^{*}\right)=V(H)$,

(ii) $I\left(H^{*}, x_{n}\right)=I\left(H, x_{n}\right), \delta\left(H^{*}, x_{n}\right)=\delta\left(H, x_{n}\right)$.

Furthermore, $H^{*}$ is symmetric about the hyperplane $x_{n}=0$ and the portion of $H^{*}$ above the hyperplane $x_{n}=0$ is a cone-like convex body $K$ as defined previously. Thus, to prove (1.6) for $n \geqq 3$ it suffices to show that for every bounded cone-like convex body $K$ with $C_{K}$ on the $x_{n}$-axis,

$$
\left[\gamma\left(K, x_{n}\right)\right]^{2} \leqq(n-1) /(n+1) .
$$

Denote by $D$ the intersection of $H^{*}$ with the hyperplane $x_{n}=0$. Then $D$ is an $(n-1)$-dimensional convex body. Since $C_{H^{*}}$ is located at the origin, the boundary of $D, \partial D$, has a representation,

$$
R=R\left(\theta_{1}, \theta_{2}, \ldots, \theta_{n-2}\right),
$$

where each $\theta_{i}, i=1,2, \ldots, n-2$, varies in the respective intervals specified in (4.3) and $R$ is a continuous strictly positive function of the indicated variables. Now, the equation (2.29) takes on the form:

$$
x_{n}=f\left(r, \theta_{1}, \theta_{2}, \ldots, \theta_{n-2}\right), \quad\left(r, \theta_{1}, \ldots, \theta_{n-2}\right) \in D .
$$

and the boundedness of $K$ implies that $f$ is (uniformly) continuous in $\bar{D}$.

Consider now the 2-dimensional flat defined by specifying a definite value for each of the coordinates, $\theta_{1}, \theta_{2}, \ldots, \theta_{n-2}$, i.e., the $\left(r, x_{n}\right)$-plane. It is a convex set because of the axiom for real numbers. Its intersection $K_{\theta}$ with the cone-like convex body $K$ is a two-dimensional convex body and it has all the properties stated in Lemma 1 . Indeed, $K_{\theta}$ is bounded from above by the curve defined by (4.5) with $\theta_{i}, i=1,2, \ldots, n-2$, being fixed. Accordingly, Lemma 2 and 3 are applicable to $K_{\theta}$. Let $T_{\theta}$ be the trapezoid constructed from $K_{\theta}$ by the method shown in Lemma 2. If we write

$$
\theta=\left(\theta_{1}, \theta_{2}, \ldots, \theta_{n-2}\right), \quad d \theta=d \theta_{1} d \theta_{2} \ldots d \theta_{n-2},
$$

then the equations (2.8) and (2.9) take on the form:

$$
\begin{aligned}
x_{n} & =x(\theta)+\alpha(\theta) r, \quad(r, \theta) \in D, \\
x(\theta) \geqq f(0, \theta) & >0, \quad x(\theta)+\alpha(\theta) R(\theta) \geqq f(R(\theta), \theta) \geqq 0,
\end{aligned}
$$


respectively, where the range of values for $\theta$ is specified in (4.3). If we transform each of the sections, $K_{\theta}$, of $K$ into a trapezoid $T_{\theta}$, then the cone-like convex body $K$ is transformed into a connected body $B$ which may not be convex. Indeed, the equations in (2.2) and (2.11) now take on the form:

$$
\begin{aligned}
r^{*}\left(K_{\theta}\right) & =m_{n-1}\left(K_{\theta}\right) / m_{n-2}\left(K_{\theta}\right), \\
x(\theta) & =n(n-1)\left[n R(\theta)-(n+1) r^{*}\left(K_{\theta}\right)\right] m_{n-2}\left(K_{\theta}\right) /[R(\theta)]^{n}, \\
\alpha(\theta) & =n(n+1)\left[n r^{*}\left(K_{\theta}\right)-(n-1) R(\theta)\right] m_{n-2}\left(K_{\theta}\right) /[R(\theta)]^{n+1} .
\end{aligned}
$$

These equations indicate that $x(\theta)$ and $\alpha(\theta)$ are uniquely determined for a given cone-like convex body $K$ and they are continuous functions of $\theta$. Thus $B$ is really a truncated cylinder with $D$ as its "base" and it is truncated from above by the "helical surface" (4.6).

From what has been said above it is now clear that

$$
\begin{aligned}
V(K) & =\int_{\theta}\left\{\int_{0}^{R(\theta)} f(r, \theta) r^{n-2} d r\right\} S(\theta) d \theta \\
V(B) & =\int_{\theta}\left\{\int_{0}^{R(\theta)}[x(\theta)+\alpha(\theta) r] r^{n-2} d r\right\} S(\theta) d \theta \\
I\left(K, x_{n}\right) & =\int_{\theta}\left\{\int_{0}^{R(\theta)} f(r, \theta) r^{n} d r\right\} S(\theta) d \theta \\
I\left(B, x_{n}\right) & =\int_{\theta}\left\{\int_{0}^{R(\theta)}[x(\theta)+\alpha(\theta) r] r^{n} d r\right\} S(\theta) d \theta \\
\delta\left(B, x_{n}\right) & =\delta\left(K, x_{n}\right)
\end{aligned}
$$

where $\theta$ stands for the region specified in (4.3) and where

$$
S(\theta) \equiv \sin ^{n-3} \theta_{1} \sin ^{n-4} \theta_{2} \ldots \sin \theta_{n-3}>0
$$

by virtue of the strict inequalities in (4.3). Thus, from the first two equations in (4.8) and the first equation in (2.10), we see that

$$
V(B)=V(K) \text {. }
$$

Also, from the third and fourth equation in (4.8), the inequality (4.9), and the inequality (2.20) with $j=1$, we see that

$$
I\left(B, x_{n}\right) \geqq I\left(K, x_{n}\right),
$$

where the equality sign holds when, and only when, $K$ and $B$ are identical bodies. Thus we conclude from (4.10), (4.11), and the last equation in (4.8) that to establish the inequality (4.4) it suffices to show that

$$
\left[\gamma\left(B, x_{n}\right)\right]^{2} \leqq(n-1) /(n+1) .
$$

To prove this inequality, we note that the value of its left-hand side remains 
unchanged if the variable $r$ is replaced by $k r$ with $k>0$. Hence, we may normalize the given convex body $H$ so that

$$
\delta\left(B, x_{n}\right)=\delta\left(K, x_{n}\right)=\delta\left(H, x_{n}\right)=1 .
$$

Upon this normalization, we can write, after rather simple but careful computation, (4.12) as follows:

$$
\begin{aligned}
\int_{\theta}\left\{[R(\theta)]^{n-1}\left[1-(R(\theta))^{2}\right]\right. & {\left.\left[\frac{1}{n+1} x(\theta)+\frac{1}{n+2} \alpha(\theta) R(\theta)\right]\right\} S(\theta) d \theta } \\
& -\frac{2}{n(n+1)(n+2)} \int_{\theta} \alpha(\theta)(R(\theta))^{n} S(\theta) d \theta \geqq 0 .
\end{aligned}
$$

Consider the value of the first integral in (4.14). We have

$$
\frac{x(\theta)}{n+1}+\frac{\alpha(\theta) R(\theta)}{n+2}=\frac{1}{n+2}[x(\theta)+\alpha(\theta) R(\theta)]+\frac{x(\theta)}{(n+1)(n+2)}>0
$$

in virtue of the inequalities in (4.7). In the meantime, the origin is the centroid of the normalized convex body $H^{*}$. Necessarily,

$$
1 \geqq R(\theta)>0 \text {. }
$$

Thus, it follows from (4.16), (4.15) and (4.9) that the value of the first integral in (4.14) is nonnegative and that it is equal to zero only when

$$
R(\theta)=1
$$

identically. That is, it vanishes only when $D$ is a unit ball of dimension $n-1$.

So far only the convexity of $K$ has been used through Lemmas 2 and 3. Now, the fact that the line $l\left(C_{K}, \mathbf{u}_{M}(K)\right)$ passes through the centroid of the cone-like convex body $K$ will be used. This will ensure that the value of the second integral in (4.14) is nonpositive. To do this we note that by virtue of $(2.30)-(2.32)$ the inequality (2.33) now takes on the form:

$$
\frac{1}{n-1} f(0, \theta) \int_{\theta}[R(\theta)]^{n-1} S(\theta) d \theta \geqq V(K)
$$

with $f(0, \theta)$ being a constant. Thus, by rather simple but careful computation, we have from the second equation in (4.8) that

$$
\begin{aligned}
V(B) & =\frac{1}{n-1} \int_{\theta} x(\theta)[R(\theta)]^{n-1} S(\theta) d \theta+\frac{1}{n} \int_{\theta} \alpha(\theta)[R(\theta)]^{n} S(\theta) d \theta \\
& =\frac{x(\hat{\theta})}{n-1} \int_{\theta}[R(\theta)]^{n-1} S(\theta) d \theta+\frac{1}{n} \int_{\theta} \alpha(\theta)[R(\theta)]^{n} S(\theta) d \theta \\
& \geqq \frac{f(0, \theta)}{n-1} \int_{\theta}[R(\theta)]^{n-1} S(\theta) d \theta+\frac{1}{n} \int_{\theta} \alpha(\theta)[R(\theta)]^{n} S(\theta) d \theta \\
& \geqq V(K)+\frac{1}{n} \int_{\theta} \alpha(\theta)[R(\theta)]^{n} S(\theta) d \theta,
\end{aligned}
$$


where the second equality follows from the mean-value theorem for integrals because $(R(\theta))^{n-1} S(\theta)>0$, the first inequality sign follows directly from the first inequality in (4.7) and the second inequality sign follows from the inequality (4.18). By combining the inequality (4.19) and the equality (4.10), we conclude that the value of the second integral in (4.14) is, indeed, nonpositive and that it is equal to zero only when both the equality sign in (4.7) and that in (4.18) hold. That is, the value of the second integral in (4.14) is nonpositive and is equal to zero when, and only when, $K$ is a right cylinder.

Since we have proved that (4.14) is a valid inequality and that the equality sign holds only when $K$ is a right circular cylinder, the inequality (1.6) for $n \geqq 3$ is now completely established.

5. Proof of the inequality (1.7) for $n>3$. To find the greatest lower bound of the radii of gyration of convex bodies with dimension $n>3$, we shall first apply McShane's splendid idea to reduce the problem in $n$-dimensional space to a problem in 2-dimensional space. Let $K$ be a given bounded convex body with dimension $n>3$. Choose $C_{K}$ as the origin of a Cartesian coordinate system such that the $x_{n}$-axis has the direction $\mathbf{u}_{m}(K)$ and that the $x_{1}$-axis meets $\partial K$ at a point with distance $\delta\left(K, C_{K}, \mathbf{u}_{m}(K)\right)$ from the origin. Denote by $K\left(x_{1}\right)$ the section of $K$ cut by the hyperplane $x_{1}=$ constant and denote by interval $[a, b]$ the projection of $K$ upon the $x_{1}$-axis. Then

$$
\begin{aligned}
I\left(K, C_{K}, \mathbf{u}_{m}(K)\right) & =\int_{a}^{b}\left\{\int_{K\left(x_{1}\right)} \sum_{i=1}^{n-1}\left(x_{i}\right)^{2} d x_{2} d x_{3} \ldots d x_{n}\right\} d x_{1} \\
& >\int_{a}^{b} x_{1}^{2}\left\{\int_{K\left(x_{1}\right)} d x_{2} d x_{3} \ldots d x_{n}\right\} d x_{1} .
\end{aligned}
$$

Denote the volume of the section $K\left(x_{1}\right)$ by

$$
A\left(x_{1}\right) \equiv \int_{K\left(x_{1}\right)} d x_{2} d x_{3} \ldots d x_{n}
$$

Then the Brunn-Minkowski theorem [5] implies that $[A(x)]^{1 /(n-1)}, a \leqq x \leqq b$, is a (continuous) concave function. That is, the Schwarz transformation is convexity preserving.

Let $\mathscr{F}$ be the family of all functions $A$ each defined continuous and nonnegative on some closed interval and having $[A(x)]^{1 /(n-1)}$ concave on that interval. For each $A(x), a \leqq x \leqq b$, in $\mathscr{F}$ we introduce the following notations:

$$
\begin{aligned}
m_{j}(A) & =\int_{a}^{b} x^{j} A(x) d x \text { for } j=0,1, \quad \bar{x}(A)=m_{1}(A) / m_{0}(A), \\
\delta(A) & =\max \{|a-\bar{x}(A)|,|b-\bar{x}(A)|\}, \\
I(A) & =\int_{a}^{b}(x-\bar{x}(A))^{2} A(x) d x, \quad g(A)=\left[I(A) / m_{0}(A)\right]^{1 / 2}
\end{aligned}
$$


Define

$$
\begin{aligned}
\mu & =\text { infimum of } \gamma\left(K, C_{K}, \mathbf{u}_{m}(K)\right) \text { for all convex bodies } K, \\
\mu^{r} & =\text { infimum of } g(A) / \delta(A) \text { for all } A \text { in } \mathscr{F} .
\end{aligned}
$$

We assert that

$$
\mu^{\prime}=\mu \text {. }
$$

To prove (5.3) we observe that if $K$ is a given bounded convex body, then $A(x)$ defined in (5.2) belongs to $\mathscr{F}$. Further,

$$
\bar{x}(A)=\bar{x}_{1}(K)=0, \quad \delta(A)=b=\delta\left(K, C_{K}, \mathbf{u}_{m}(K)\right) .
$$

Hence (5.1) implies that

$$
g(A) / \delta(A)<\gamma\left(K, C_{K}, \mathbf{u}_{m}(K)\right) .
$$

This shows that $\mu^{\prime} \leqq \mu$. On the other hand, let $A(x), a \leqq x \leqq b$, belong to $\mathscr{F}$. For every positive number $\varepsilon$, let $K_{\varepsilon}$ be the body consisting of those points $\left(x_{1}, x_{2}, \ldots\right.$, $x_{n}$ ) such that

$$
x_{1} \in[a, b], \quad \sum_{i=2}^{n}\left(x_{i}\right)^{2} \leqq \varepsilon\left[A(x) / \lambda_{n-1}\right]^{1 /(n-1)},
$$

where $\lambda_{n-1}$ stands for the volume of a unit ball of dimension $n-1$. Then $K_{\varepsilon}$ is convex and its centroid is at $(\bar{x}(A), 0, \ldots, 0)$. Let $l$ be the line through the centroid of $K_{\varepsilon}$ and parallel to the $x_{n}$-axis. Then

$$
\begin{gathered}
V\left(K_{\varepsilon}\right)=\int_{a}^{b} \varepsilon^{n-1} A\left(x_{1}\right) d x_{1}, \\
I\left(K_{\varepsilon}, l\right)=\int_{a}^{b}\left[\left(x_{1}-\bar{x}(A)\right)^{2}+\varepsilon^{2}\left[A\left(x_{1}\right) / \lambda_{n-1}\right]^{2 /(n-1)}\right] \varepsilon^{n-1} A\left(x_{1}\right) d x_{1} .
\end{gathered}
$$

Thus

$$
\lim _{\varepsilon \rightarrow 0} g\left(K_{\varepsilon}, l\right)=\left[\int_{a}^{b}\left(x_{1}-\bar{x}(A)\right)^{2} A\left(x_{1}\right) d x_{1} / \int_{a}^{b} A\left(x_{1}\right) d x_{1}\right]^{1 / 2}=g(A) .
$$

Also, from (5.4) it is easy to see that $\delta\left(K_{\varepsilon}, l\right)$ tends to $\delta(A)$ as $\varepsilon \rightarrow 0$. Therefore

$$
\lim _{\varepsilon \rightarrow 0}\left[g\left(K_{\varepsilon}, l\right) / \delta\left(K_{\varepsilon}, l\right)\right]=g(A) / \delta(A) .
$$

This shows that $\mu \leqq \mu^{\prime}$ and hence the assertion (5.3) is proved.

The above reasoning shows that to establish inequality (1.7) it suffices to show

$$
\mu^{\prime}=1 / n(n+2) \text {. }
$$

One might follow McShane's technique to prove (5.5) by negation [1]. We would like to present a variant proof by comparison method. Let $A(x)$ be a given function in $\mathscr{F}$. Without loss of generality we may assume that it is defined on $[0,1]$ and 
that $\bar{x}(A) \leqq 1 / 2$. Since the convex body $K_{\varepsilon}$ constructed from $A(x)$ by the rules in (5.4) has $(\bar{x}(A), 0, \ldots, 0)$ as its centroid, it follows that [7]

$$
1 /(n+1) \leqq \bar{x}(A) \leqq 1 / 2 .
$$

We proceed to construct a specific function $A^{*}(x)$ in $\mathscr{F}$ such that

$$
g\left(A^{*}\right) / \delta\left(A^{*}\right) \leqq g(A) / \delta(A)
$$

Consider the function

$$
\begin{aligned}
A^{*}(x) & =[\eta x / \xi]^{n-1}, \quad 0 \leqq x \leqq \xi, \\
& =[\eta(1-x) /(1-\xi)]^{n-1}, \quad \xi \leqq x \leqq 1 .
\end{aligned}
$$

Clearly, $A^{*}$ belongs to $\mathscr{F}$. Furthermore, direct computation yields:

$$
\begin{gathered}
m_{0}\left(A^{*}\right)=\eta / n, \\
1 / n(n+1) \leqq \bar{x}\left(A^{*}\right)=[1+(n-1) \xi] / n(n+1) \leqq 1 /(n+1) .
\end{gathered}
$$

These two relations together with that in $(5.6)$ indicate that $(\xi, \eta)$ can be so chosen that

$$
m_{0}\left(A^{*}\right)=m_{0}(A), 1-\delta\left(A^{*}\right)=\bar{x}\left(A^{*}\right)=\bar{x}(A)=1-\delta(A) .
$$

If the functions $A$ and $A^{*}$ are identical, then the inequality (5.7) is trivially valid. Accordingly we assume that this is not the case. In order to compare $I\left(A^{*}\right)$ with $I(A)$ we consider the following two pairs of curves:

$$
\begin{gathered}
y=A(x), \quad y=A^{*}(x), \quad 0 \leqq x \leqq 1 ; \\
y=[A(x)]^{1 /(n-1)}, \quad y=\left[A^{*}(x)\right]^{1 /(n-1)}, \quad 0 \leqq x \leqq 1 .
\end{gathered}
$$

It is useful to observe that the points of intersection of the two curves in (5.10) are also the intersection points of those two in (5.11) and conversely. Furthermore, one of the curves in (5.11) is concave downward, while the other consists of two line segments. From these facts and the two requirements in (5.9), we conclude, by the same reasoning as for Lemma 2 , that the two curves in (5.10) intersect at two, and only two, points over the interval $[0,1]$ and that

$$
\eta^{n-1} \geqq \max \{A(x) ; x \in[0,1]\}, \quad A^{*}(0) \leqq A(0), \quad A^{*}(1) \leqq A(1),
$$

where any one of the equality signs holds when, and only when, $A^{*}$ and $A$ are identical. Moreover, by the same reasoning as for Lemma 3, we conclude from (5.12) that

$$
\int_{0}^{1} x^{2} A^{*}(x) d x \leqq \int_{0}^{1} x^{2} A(x) d x
$$

where the equality sign holds only when $A^{*}$ and $A$ are identical. This inequality together with the parallel-axis theorem for moment of inertia ensures that 
$I\left(A^{*}\right) \leqq I(A)$ and they are equal only when $A^{*}$ and $A$ are identical. Hence, the inequality (5.7) is, indeed, satisfied by the function $A^{*}(x)$ in (5.8) provided the two conditions in (5.9) hold.

To complete the proof for (1.7) it remains to show that

$$
\left[g\left(A^{*}\right) / \delta\left(A^{*}\right)\right]^{2} \geqq 1 / n(n+2) .
$$

But $A^{*}(x ; \xi)$ is an elementary function in $x$ and the parameter $\xi$. Direct computation shows that $n(n+2)\left[g\left(A^{*}\right)\right]^{2}-\left[\delta\left(A^{*}\right)\right]^{2}$ is a positive constant multiple of

$$
\left(5 n^{4}+6 n^{3}+5 n^{2}\right) \xi(1-\xi)+\left(n^{4}+6 n^{3}+n^{2}-\xi\right) \xi .
$$

Since, for $\bar{x}\left(A^{*}\right) \leqq 1 / 2$, it is necessary that $0 \leqq \xi \leqq 1 / 2$, the expression in (5.14) proves that (5.13) is a valid inequality and that the equality sign there holds only when $\xi=0$. The inequality (1.7) is now established.

6. A generalized McShane problem. In the proof of the theorem, only partial results of Lemma 3 have been used. Motivated by this fact, we proceed to formulate a generalized McShane problem. Consider a bounded convex body $K$ with dimension $n>1$. For each integer $j \geqq 2$, we denote by $I_{j}\left(K, C_{K}, \mathbf{u}\right)$ and $g_{j}\left(K, C_{K}, \mathbf{u}\right)$ respectively the $j$ th moment of inertia and the $j$ th radius of gyration of $K$ about the line $l\left(C_{K}, \mathbf{u}\right)$ and define them by the following formulas:

$$
\begin{aligned}
I_{j}\left(K, C_{K}, \mathbf{u}\right) & \equiv \int_{K}\left[\rho\left(X ; l\left(C_{K}, \mathbf{u}\right)\right)\right]^{j} d X, \\
g_{j}\left(K, C_{K}, \mathbf{u}\right) & \equiv\left[I_{j}\left(K, C_{K}, \mathbf{u}\right) / V(K)\right]^{1 / j} .
\end{aligned}
$$

Similarly, the $j$ th relative radius of gyration of $K$ about $l$ is defined by

$$
\gamma_{j}\left(K, C_{K}, \mathbf{u}\right)=g_{j}\left(K, C_{K}, \mathbf{u}\right) / \delta\left(K, C_{K}, \mathbf{u}\right) .
$$

For a given bounded convex body $K$ and for a given integer $j \geqq 2$, it is clear that there are two directions $\mathbf{u}_{M}(K, j), \mathbf{u}_{m}(K, j)$ such that

$$
\begin{aligned}
& \gamma_{j}\left(K, C_{K}, \mathbf{u}_{M}(K, j)\right)=\text { maximum of } \gamma_{j}\left(K, C_{K}, \mathbf{u}\right) \text { for all } \mathbf{u}, \\
& \gamma_{j}\left(K, C_{K}, \mathbf{u}_{m}(K, j)\right)=\text { minimum of } \gamma_{j}\left(K, C_{K}, \mathbf{u}\right) \text { for all } \mathbf{u} .
\end{aligned}
$$

We generalize McShane's problem by asking for the greatest lower bounds and the least upper bounds for the relative radii of gyration of order $j \geqq 2$ among the class of all bounded convex bodies.

To determine the least upper bounds, we need only to strengthen Lemma 3 as follows:

Lemma 3*. Let $K$ be the convex set specified in Lemma 1. For a given integer $n \geqq 2$ and for the given set $K$, let $T$ be the trapezoid constructed in Lemma 2. Then

$$
m_{n-1+j}(T) \geqq m_{n-1+j}(K) \quad \text { for } j=1,2, \ldots,
$$

where any one of the equality signs holds only when $T=K$. 
Proof. We shall use the same notation as adopted in Lemma 1, 2 and 3. For simplicity we write

$$
\begin{gathered}
g(x) \equiv y_{0}+\alpha x-f(x), \quad 0 \leqq x \leqq x_{1}, \\
m(g ; n-1+j) \equiv m_{n-1+j}(T)-m_{n-1+j}(K) .
\end{gathered}
$$

By the very method for constructing $T$, we have

$$
m(g ; n-2)=0, \quad m(g ; n-1)=0 .
$$

Hence there are two constants, $x_{3}, x_{3,0}$ such that

$$
\begin{array}{rlrl}
x_{4} & <x_{3,0}<x_{3}<x_{2}, \\
\int_{0}^{x_{3}} x^{n-2} g(x) d x & =0, & & \int_{x_{3}}^{x_{1}} x^{n-2} g(x) d x=0, \\
\int_{0}^{x_{3,0}} x^{n-1} g(x) d x & =0, & & \int_{x_{3,0}}^{x_{1}} x^{n-1} g(x) d x=0 .
\end{array}
$$

For the constant $x_{3,0}$ we have from the mean-value theorem that

$$
\int_{0}^{x_{3,0}} x^{(n-1)+1} g(x) d x=C_{4} \int_{0}^{x_{4}} x^{n-1} g(x) d x+C_{3} \int_{x_{4}}^{x_{3,0}} x^{n-1} g(x) d x
$$

with

$$
0<C_{4}<x_{4}<C_{3}<x_{3,0} \text {. }
$$

Hence it follows from (6.2), (6.3), (2.21) that the value of the integral in (6.3) is negative. Accordingly, in view of (2.21) there is a constant $x_{3,1}$ such that

$$
\begin{gathered}
x_{4}<x_{3,1}<x_{3,0}<x_{2}, \\
\int_{0}^{x_{3,1}} x^{(n-1)+1} g(x) d x=0, \quad \int_{x_{3,1}}^{x_{1}} x^{(n-1)+1} g(x) d x \geqq 0,
\end{gathered}
$$

because it has been proved that $m(g ; n-1+1) \geqq 0$. On the other hand we have, by the mean-value theorem for integrals,

$$
\begin{aligned}
\int_{0}^{x_{1}}\left(x-x_{3,1}\right)^{2} x^{n-1} g(x) d x & =\left[\left(c_{4}-x_{3,1}\right)^{2} \int_{0}^{x_{4}}+\left(c_{3}-x_{3,1}\right)^{2} \int_{x_{4}}^{x_{3,1}}\right. \\
& \left.+\left(c_{2}-x_{3,1}\right)^{2} \int_{x_{3,1}}^{x_{2}}+\left(c_{1}-x_{3,1}\right)^{2} \int_{x_{2}}^{x_{1}}\right]\left[x^{n-1} g(x)\right] d x,
\end{aligned}
$$

with

$$
0<c_{4}<x_{4}<c_{3}<x_{3,1}<c_{2}<x_{2}<c_{1}<x_{1} .
$$

Hence it follows from (6.4)-(6.6), the last two equations in (6.2) and the inequality (2.21) that

$$
\int_{0}^{x_{1}}\left(x-x_{3,1}\right)^{2} x^{n-1} g(x) d x \geqq 0
$$


and that it is equal to zero only when $K=T$. The inequality (6.7) can be written as

$$
m(g ; n-1+2)-2 x_{3,1} m(g ; n-1+1)+x_{3,1}^{2} m(g ; n-1) \geqq 0 .
$$

In view of $(6.1)$ and the fact that $m(g ; n-1+1) \geqq 0$, it follows from (6.8) that

$$
\begin{aligned}
m(g ; n-1+2)-x_{3,1} m & (g ; n-1+1) \\
& \geqq x_{3,1}\left[m(g ; n-1+1)-x_{3,1} m(g ; n-1)\right] \geqq 0 .
\end{aligned}
$$

With the suggestions of the results obtained above we wish to show that for each integer $j \geqq 1$ there is a constant $x_{3, j}$ such that

(i) $x_{4}<x_{3, j}<x_{3, j-1}<x_{2}$,

(ii) $\int_{0}^{x_{3.5}} x^{(n-1)+j} g(x) d x=0, \quad \int_{x_{3}, j}^{x_{1}} x^{(n-1)+j} g(x) d x \geqq 0$,

(iii) $m(g ; n-1+j+1)-x_{3, j} m(g ; n-1+j) \geqq 0$,

where the equality sign holds only when $K=T$.

Our proof for the above statement is by induction on the integer $j$. The relations in (6.4)-(6.9) indicate that the statement is true for $j=1$. It remains to show that if the statement is true for integer $j$ then it is also true for integer $j+1$. By the induction hypothesis, there is a constant, $x_{3, j}$, such that all the relations in (i), (ii) and (iii) hold for the integer $j$. Since for this constant $x_{3, j}$

$$
\int_{0}^{x_{3.1}} x^{(n-1)+(j+1)} g(x) d x=c_{4} \int_{0}^{x_{4}} x^{(n-1)+j} g(x) d x+x_{3} \int_{x_{4}}^{x_{3 . j}} x^{(n-1)+j} g(x) d x
$$

with

$$
0<c_{4}<x_{4}<c_{3}<c_{3, j}<x_{2}
$$

its value must be negative in view of the first equation in (ii) and the inequalities in (2.21). But as a consequence of (iii) and the induction hypothesis,

$$
m(g ; n-1+k) \geqq 0 \quad \text { for } k=1,2, \ldots, j .
$$

Hence, in view of the inequalities in (2.21) there is a constant, $x_{3, j+1}$, such that

(a) $x_{4}<x_{3, j+1}<x_{3, j}<x_{2}$,

(b) $\int_{0}^{x_{3, j+1}} x^{(n-1)+(j+1)} g(x) d x=0, \quad \int_{x_{3, j+1}}^{x_{1}} x^{(n-1)+(j+1)} g(x) d x \geqq 0$.

Furthermore, by the same argument as shown in (6.5); (6.6), we have

$$
\int_{0}^{x_{1}}\left[x-x_{3, j+1}\right]^{2} x^{(n-1)+j} g(x) d x \geqq 0 .
$$

This means that

$$
\begin{aligned}
m(g ; n-1+j+2)-x_{3, j+1} m(g ; n-1+j+1) & \\
& \geqq x_{3, j+1}\left[m(g ; n-1+j+1)-x_{3, j+1} m(g ; n-1+j)\right] .
\end{aligned}
$$

But the inequalities in (a) and the induction hypothesis (iii) assure that the righthand side in (6.10) is nonnegative. Hence

(c) $m(g ; n-1+j+2)-x_{3, j+1} m(g ; n-1+j+1) \geqq 0$. 
The results in (a), (b), and (c) state that (i), (ii) and (iii) also hold for integer $j+1$. Our statement is now proved and hence the lemma is established.

Using Lemma $3^{*}$ and following the same procedure as shown in $\$ 4$, one can show that for all bounded convex bodies $K$ with dimension $n>1$,

$$
\left[\gamma_{j}\left(K, C_{K}, \mathbf{u}_{M}(k, j)\right)\right]^{j} \leqq(n-1) /(n+j-1),
$$

where the equality sign holds only when $K$ is a right circular cylinder. We leave the question open as to how to determine the greatest lower bounds for the relative radii of gyration of all finite orders.

\section{REFERENCES}

1. E. J. McShane, The radius of gyration of a convex body, Proc. Amer. Math. Soc. 13 (1962), 922-926.

2. T. W. Ting, An isoperimetric inequality for moments of inertia of plane convex sets, Trans. Amer. Math. Soc. 107 (1963), 421-431.

3. P. R. Garabedian, Partial differential equations, Wiley, New York, 1964; Chapters 11, 15.

4. G. Pólya and G. Szegö, Isoperimetric inequalities in mathematical physics, Annals of Mathematics Studies No. 27, Princeton Univ. Press, Princeton, N. J., 1951; pp. 151-157, 182-186.

5. T. Bonnesen and W. Fenchel, Theorie der konvexer Körper, Chelsea, New York, 1948; pp. 69-73.

6. J. L. W. V. Jensen, Sur les fonctions convexes et les inégalités entre les valeurs moyennes, Acta Math. 30 (1906), 175-193.

7. P. C. Hammer, Centroids of convex bodies, Proc. Amer. Math. Soc. 2 (1951), 522-525.

8. G. H. Hardy, J. E. Littlewood, and G. Pólya, Inequalities, Cambridge Univ. Press, Cambridge, 1934; pp. 71-81.

North Carolina State University,

Raleigh, North Carolina 